\title{
SOLUTIONS OF PROBLEMS OF MILLER AND RUBEL
}

\section{SAKARI TOPPILA}

1. Introduction and results. Let $w$ be analytic in $|z|<1$ with $w(0)=0$. In Problem 5.61 of [2] S. Miller states that if $n=1$ or $n=2$ and

$$
\left|w(z)+z w^{\prime}(z)+\ldots+z^{n} w^{(n)}(z)\right|<1 \text { for }|z|<1,
$$

then $|w(z)|<1$ for $|z|<1$, and he asks whether the same holds for all $n=1,2,3, \ldots$. We shall give an affirmative answer to this question.

Theorem 1. Let $w$ be as above, satisfying (1.1) for some $n \geqq 3$. Then $|w(z)|<$ $71 / 80$ in $|z|<1$.

The following problem [1, Problem 2.54] is proposed by L. A. Rubel.

Problem A. Let $E$ be a closed set in $C$ with the following properties: (1) there exists a transcendental entire function $f(z)$ that is bounded on $E$; and (2) there exists a transcendental entire function $g(z)$ that is bounded away from 0 on the complement of E. For each such E, must there exist one transcendental entire function that is simultaneously bounded on $E$ and bounded away from 0 on the complement of $E$ ?

We shall give the following answer to this question.

Theorem 2. Let $E$ be the union of the positive real axis and the closed discs $\left|z-e^{4 k}\right| \leqq 1, \quad k=1,2, \ldots$. Then there exist transcendental entire functions $f_{1}$ and $f_{2}$ such that $f_{1}$ is bounded on $E$ and that $f_{2}$ is bounded away from 0 on the complement of $E$. However, if $f$ is any entire function which is bounded on $E$ and bounded away from 0 on the complement of $E$, then $f$ is constant.

2. Proof of Theorem 1. Let $n$ and

be as in Theorem 1, and let

$$
w(z)=\sum_{p=1}^{\infty} a_{p} z^{p}
$$

$$
g(z)=w(z)+z w^{\prime}(z)+\ldots+z^{n} w^{(n)}(z)=\sum_{p=1}^{\infty} b_{p} z^{p} .
$$

Since

$$
w^{(k)}(z)=\sum_{p=1}^{\infty} a_{p} p(p-1) \ldots(p-k+1) z^{p-k}
$$


and $n \geqq 3$, we get $b_{1}=2 a_{1}, b_{2}=5 a_{2}, b_{3}=16 a_{3}$, and

$$
\left|b_{p}\right| \geqq\left|a_{p}(1+p+(p-1) p+(p-2)(p-1) p)\right| \geqq\left|a_{p}\right|(p-1)^{3}
$$

for $p \geqq 4$. From (1.1) we deduce that $\left|b_{p}\right| \leqq 1$ for any $p$, and we get

$$
\sum_{p=1}^{\infty}\left|a_{p}\right| \leqq 1 / 2+1 / 5+1 / 16+\sum_{p=3}^{\infty} p^{-3}<61 / 80+\int_{2}^{\infty} x^{-3} d x=71 / 80,
$$

which implies that $|w(z)|<71 / 80$ in $|z|<1$. Theorem 1 is proved.

3. Proof of Theorem 2. The function $f_{1}(z)=e^{-z}$ is bounded on $E$ and

$$
f_{2}(z)=\prod_{k=1}^{\infty}\left(1-z e^{-4 k}\right)
$$

is bounded away from 0 on the complement of $E$.

Let us suppose that $f$ is an entire function which satisfies $|f(z)| \leqq M$ on $E$ and $|f(z)| \geqq m(m>0)$ on the complement of $E$. From the continuity of $f$ it follows that $|f(z)| \geqq m$ on the ring domain

$$
e^{4 k+1}<|z|<e^{4 k+3}
$$

for any $k$. Since $e^{4 k+2} \in E$, we deduce that $\left|f\left(e^{4 k+2}\right)\right| \leqq M$, and applying Schottky's theorem repeatedly in the discs

$$
\left|z-e^{4 k+2} e^{i \alpha}\right|<e^{4 k+2} / 2,
$$

we deduce that there exists $K>0$ depending only on $M$ and $m$ such that $|f(z)| \leqq K$ for all $z$ lying on the circle $|z|=e^{4 k+2}, k=1,2, \ldots$. This implies that $f$ is a bounded function and we deduce that $f$ is constant. Theorem 2 is proved.

\section{References}

[1] Anderson, J. M., K. F. Barth, and D. A. Brannan: Research problems in complex analysis. Bull. London Math. Soc. 9, 1977, 129-162.

[2] Campbell, D. M., J. G. Clunie, and W. K. Hayman: Research problems in complex analysis. Aspects of contemporary complex analysis (Proceedings of an Instructional Conference organized by the London Mathematical Society at the Univetsity of Durham, Durham 1979) edited by D. A. Brannan and J. G. Clunie, Academic Press, London-New York-Toronto-Sydney-San Francisco, 1980, 527-572.

University of Helsinki

Department of Mathematics

SF-00100 Helsinki 10

Finland

Received 25 May 1983 